\section{Hemoptysis Due to Breath-Hold Diving Following Chemotherapy and Lung Irradiation: A Differential Diagnosis; with Authors' Reply}

Editor - In a recent case report published in your journal, a 60-year-old male breath-hold diver was reported to have experienced hemoptysis after five repetitive apnea dives to a maximum depth of 13 meters of sea water (MSW). ${ }^{1}$ The patient's past medical history included treatment for breast carcinoma with surgery followed by adjuvant chemo- and radiotherapy. The radiotherapy had resulted in parenchymal fibrosis in the irradiated part of the lung. It was concluded by the authors that the patient most likely suffered from alveolar hemorrhage due to capillary stress failure. As explained in more detail in the discussion by the authors, this occurs when abnormally high stress in the walls of the pulmonary capillaries leads to ultra-structural changes such as disruption of the capillary and alveolar endothelium, resulting in pulmonary edema and potentially pulmonary hemorrhage. The authors also stated that pulmonary barotrauma (PBT) was less likely because of the shallow diving depth. However, in our opinion, PBT is a plausible pathophysiological mechanism in this case. We would like to highlight the pathophysiological mechanism that could have precipitated PBT in this case and emphasize the notion that diving at relatively shallow depths is not protective for barotrauma.

PBT occurs when a pressure gradient across the alveolar membrane develops that exceeds its breaking point. When lung volume adapts to changes in absolute pressure in accordance with Boyle's law, no significant gradient develops during descent or ascent. However, a critical prerequisite for accommodation of volume/pressure changes in the lung during a dive is the absence of structural lung abnormalities that compromise lung tissue compliance. ${ }^{2}$ Healthy lung tissue (schematic model in figure 1, left panel) can accommodate for the increased pressure at depth by allowing for a decrease in volume, preventing a pressure gradient across the alveolar wall. In contrast, when the lung tissue is characterized by decreased compliance due to inflammation or fibrosis (figure 1 , right panel), the alveolar space cannot adapt its volume in proportion to the increased pressure, and a pressure gradient builds up.

It is a common misconception that diving depths of $10 \mathrm{MSW}$, for example, can be considered as being shallow and unlikely to cause PBT. In fact, as shown in figure 2, the absolute and relative changes in gas volume across diving depths is greatest at shallower depths due to the exponential relationship between volume and pressure. The minimal pressure gradient required to cause rupture of the lung membrane can be reached with a pressure equivalent to only $1 \mathrm{MSW}^{3}$ Several cases of PBT have been reported to occur in relation to breath-hold dives at depths not exceeding $5 \mathrm{MSW}^{4-6}$

Obstruction may be an additional risk factor for PBT when redistribution of air to peripheral alveoli occurs in lung areas with interstitial fibrosis and air way obstruction, such as was possibly present in the reported patient. Air trapping may lead to over-inflation trauma during ascent. Although mostly a risk in divers breathing compressed air, overexpansion injury during ascent has also been reported in breath-hold divers. ${ }^{7}$

It remains difficult to assess the mechanism of alveolar damage in the reported case. The absence of free air on the computed tomography (CT) scan does not rule out PBT, as PBT can be difficult to detect, depending on the size of the defect, the presence of other intrapulmonary pathology, and the time period after which a CT scan is obtained. The pivotal role of the pre-existing lung disease in this case is stressed by the fact that the alveolar hemorrhage occurred in the irradiated areas of the lung where parenchymal abnormalities were chronically present. The tearing effects from pressure gradients formed between peribronchiolar and perialveolar
Healthy

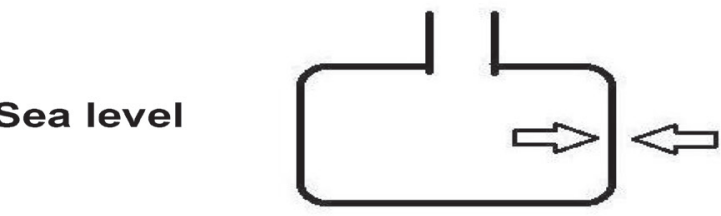

Depth

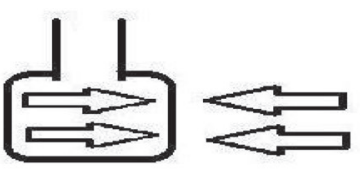

\section{Parenchymal lung disease}
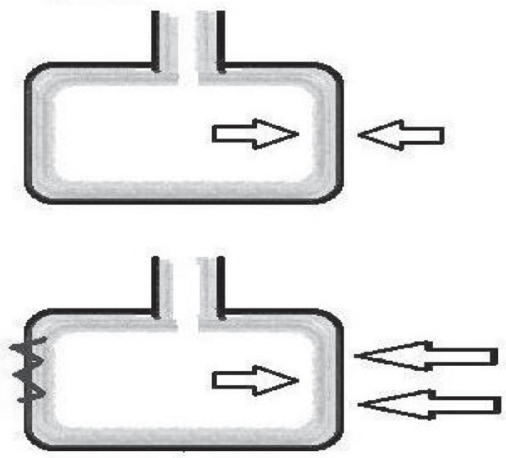

Figure 1. Pathophysiological model of the influence of parenchymal lung disease on the development of an alveolar membrane pressure gradient. 


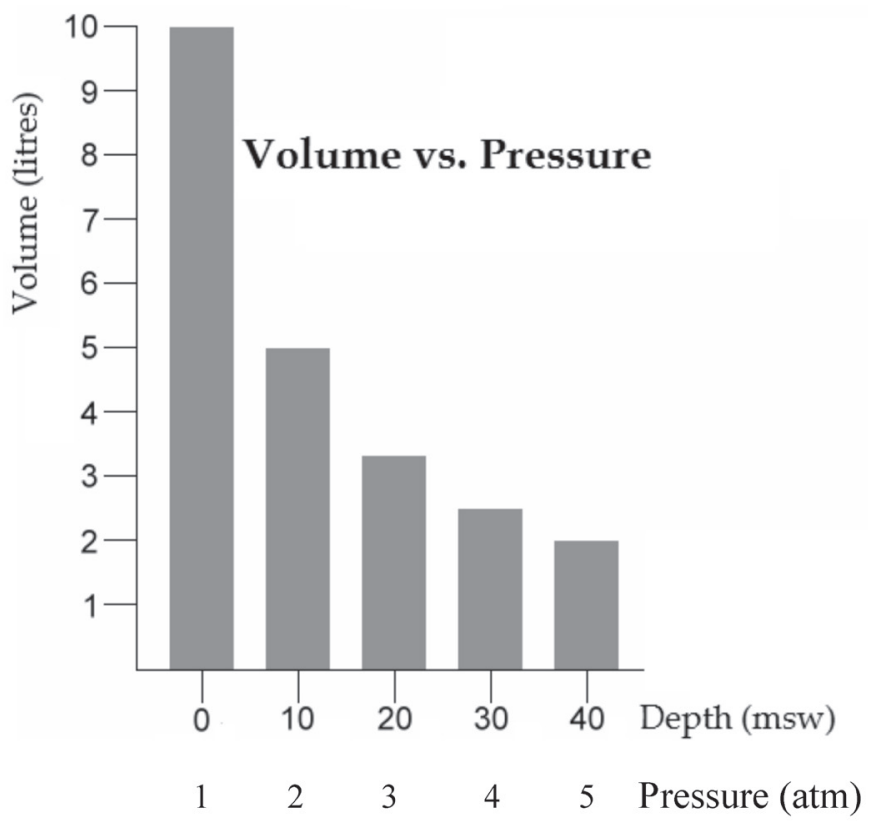

Figure 2. Volume changes in relation to diving depth.

tissues during gas-expansion tend to be greater if the elasticity of different lung areas is inhomogeneous. ${ }^{2}$

In conclusion, we agree with the authors that capillary stress failure is a plausible diagnosis in the reported case. However, PBT needs to be considered as a differential diagnosis. Divers with a history of pulmonary disease or thoracic irradiation need to be screened for interstitial lung disease by spirometry and CT of the thorax to exclude parenchymal pulmonary disease that may pose a risk of PBT during both Scuba and breath-hold diving.

\section{References}

1. Gutsche M, Kuschner WG. Hemoptysis due to breath-hold diving following chemotherapy and lung irradiation. Clin Med Res 2012;10:137-139.

2. Francis J. Pulmonary barotrauma: a new look at mechanisms. SPUMS Journal 1997;27:205-218.
3. Malhotra MS, Wright HC. The effects of a raised intrapulmonary pressure on the lungs of fresh unchilled cadavers. J Pathol Bacteriol 1961;82:198-202.

4. Bayne CG, Wurzbacher T. Can pulmonary barotrauma cause cerebral air embolism in a non-diver? Chest 1982;81: 648-650.

5. Perez J, Teatino R. Pneumomediastinum in a snorkelling diver . BMJ Case Rep 2012;2012:pii. doi: 10.1136/bcr.09.2011.4776

6. Henckes A, Arvieux J, Cochard G, Jézéquel P, Arvieux CC. Hemoptysis and pneumomediastinum after breath-hold diving in shallow water: a case report. Undersea Hyperb Med 2011;38:213-216.

7. Kol S, Weisz G, Melamed Y. Pulmonary barotrauma after a free dive--a possible mechanism. Aviat Space Environ Med 1993;64:236-237.

Valerie A. Fijen, MSc

Junior research associate on the Diving with Disorders in

Hemostasis (DIDIH) research project from the Dutch

Society for Diving Medicine

Diving Medical Center, Royal Netherlands Navy,

Den Helder

Peter E. Westerweel, $M D, P h D$

Internist- hematologist, Albert Schweitzer Hospital,

Dordrecht, the Netherlands;

Diving physician with the Dutch Society for Diving

Medicine

Robert A. van Hulst, $M D, P h D$

Director Diving Medical Center, Royal Netherlands Navy,

Den Helder

Head Research Hyperbaric and Diving Medicine, AMC,

Amsterdam

Diving physician with the Dutch Society for Diving

Medicine

Received: April 30, 2013

Accepted: May 9, 2013

doi: $10.3121 / \mathrm{cmr} .2013 .1173-8$

Markus Gutsche, $M D, P h D$

Pulmonary Section, US Department of Veterans Affairs Palo Alto Health Care System, Palo Alto, California, USA; and Division of Pulmonary and Critical Care Medicine, Stanford University School of Medicine, Stanford, California, USA

Ware Kuschner, MD

Chief, Pulmonary Section, US Department of Veterans Affairs Palo Alto Health Care System, Palo Alto, California, USA; and Division of Pulmonary and Critical Care Medicine, Stanford University School of Medicine, Stanford, California, USA

Received: May 16, 2103

Accepted: May 23, 2013

doi: $10.3121 / \mathrm{cmr} .2013 .1173-8-\mathrm{a}$ 